\title{
Reología del yogur: efectos de las operaciones unitarias en el procesamiento y uso de aditivos
}

\section{Yogurt rheology: effects of unit operations on processing and use of additives}

\author{
Rosa Mendoza ${ }^{*} *$, Sebastián Guerrero ${ }^{(0)}$, Byron Herrera-Chávez \\ Carrera de Agroindustrial, Facultad de Ingeniería, Universidad Nacional de Chimborazo, Riobamba, Ecuador, 060150; \\ saguerrero@unach.edu.ec; bherrera@unach.edu.ec \\ * Correspondencia: remendoza.fiag@unach.edu.ec
}

\begin{abstract}
Citación: Mendoza, R., Guerrero, S., \& Herrera-Chávez, B. (2021). Reología del yogur: efectos de las operaciones unitarias en el procesamiento y uso de aditivos. Novasinergia. 4(1). 151-163. https://doi.org/10.37135/ns.01.07. 09
\end{abstract}

Recibido: 25 septiembre 2020 Aceptado: 23 noviembre 2020 Publicado: 01 junio 2021

Novasinergia ISSN: 2631-2654
Resumen: El objetivo de esta investigación es determinar los factores que influyen en las características reológicas del yogur batido, mediante una revisión sistemática de artículos científicos utilizando el método PRISMA para la inclusión y exclusión de información. Para lograr nuestro objetivo se analizaron las variables relacionadas a las operaciones unitarias como la estandarización, pasteurización, inoculación, batido y almacenamiento que afectan a la viscosidad del yogur batido, estableciendo (temperaturas, tiempos y velocidades para cada operación unitaria) basados en la información proporcionada en los artículos consultados. Además, se analizó los aditivos que afectan a la viscosidad del yogur tomando en cuenta las características de la muestra analizada, el equipo utilizado y velocidades de corte, determinando que todos estos factores afectaban significativamente la viscosidad del yogur. El análisis realizado permite establecer que la viscosidad es influenciada por las características físico-químicas de la leche y operaciones unitarias presentes en el procesamiento de yogur. Los emulsionantes y estabilizantes influyen directamente en la viscosidad del producto final contribuyendo al aumento de la sinéresis durante el tiempo de almacenamiento.

Palabras clave: Biotecnología de alimentos, emulsificantes del yogur, estabilizantes del yogur, ingeniería de alimentos, productos lácteos, yogur batido, reología del yogur, tecnología de alimentos, viscosidad del yogur.

\begin{abstract}
This research aims to determine the factors that influence stirred yogurt's rheological characteristics through a systematic review of scientific articles using the PRISMA method to exclude and include information. We analyzed the variables related to unit operations such as standardization, pasteurization, inoculation, stirred, and storage to achieve our objective. These variables affect stirred yogurt's viscosity, establishing (temperatures, times, and speeds for each unit operation) based on the information provided in the articles consulted. Also, the additives that affect the yogurt's viscosity were analyzed, taking into account the characteristics of the sample analyzed, the equipment used, and cutting speeds, determining that all these factors significantly affect the viscosity of the yogurt. The analysis carried out allows establishing that the viscosity is influenced by the physicochemical characteristics of the milk and unit operations present in yogurt processing. Emulsifiers and stabilizers affect the final product's viscosity and increase the syneresis during storage time.
\end{abstract}

Keywords: Dairy products, food biotechnology, food engineering, food technology, stirred yogurt, yogurt emulsifiers, yogurt rheology, yogurt stabilizers, yogurt viscosity. 


\section{Introducción}

Actualmente se consumen leches fermentadas por sus múltiples beneficios y por sus propiedades organolépticas agradables al consumidor, siendo el yogur uno de los alimentos lácteos más apetecidos a nivel mundial (O'Donnell \& Butler, 2002). El yogur es un fluido no newtoniano, que presenta un adelgazamiento por cizallamiento, su viscosidad cambia cuando el gradiente de velocidad varía y posee un comportamiento de flujo tixotrópico -depende del tiempo y esfuerzo cortante- (Afonso \& Maia, 1999).

Las propiedades reológicas del yogur son importantes en el diseño de procesos de flujo, en su control de calidad, procesamiento y almacenamiento, y en la predicción de la consistencia del yogur (Soukoulis, Panagiotidis, Koureli, \& Tzia, 2007). Uno de los parámetros fundamentales que caracterizan el comportamiento de flujo en alimentos semilíquidos es la viscosidad, que actualmente se ha considerado uno de los principales atributos de calidad para determinar la aceptabilidad por el consumidor (Wu et al., 2009). En la industria láctea el yogur al ser un material viscoelástico puede presentar defectos en las variaciones de la viscosidad (falta de fuerza del gel) y en la separación del suero (sinéresis), como consecuencia de un cambio en la composición química de la leche, temperatura de incubación excesiva o heterogénea, diferentes tipos de cultivo sembrados, temperatura de enfriamiento inadecuada y falta de cuidado en el manejo del gel (Molina, 2009).

Los problemas relacionados con alteraciones en las características físicas y sensoriales del yogurt son más, que los originados por contaminación microbiológica (Vélez-Ruiz, Barbosa, \& Peleg, 1997). El coágulo de yogur a menudo se somete a agitación, lo que genera que sea menos viscoso o en casos extremos, puede muestre separación de suero (Tamime, Davies, Chehade, \& Mahdi, 1989). Los estabilizantes, como la pectina o la gelatina, a menudo se agregan a la base de la leche para mejorar o mantener las propiedades apropiadas del yogur (viscosidad/consistencia) para la prevención de la separación del suero (Tamime, Muir, \& Wszolek, 1999). El uso de estabilizantes puede ayudar a proporcionar una consistencia más uniforme. Sin embargo, puede haber defectos de textura relacionados con el uso de estabilizantes, incluida la estabilización excesiva (sobre estabilización) que da como resultado un yogur elástico "gelatinoso", lo que puede generar una separación débil del cuerpo o del suero "líquida" (Vedamuthu, 1991), lo que ocasionan un efecto negativo en las propiedades físicas y sensoriales del producto (Guénard-Lampron, St-Gelais, Villeneuve, \& Turgéon, 2019).

El objetivo de esta investigación fue realizar una revisión bibliográfica de los factores que influyen en las características reológicas del yogur batido, debido a que el mismo presenta un comportamiento de flujo complejo, dependiente del esfuerzo cortante y del tiempo. La importancia del estudio de la reología radica en que no existen parámetros estandarizados relacionados con las temperaturas de incubación, enfriamiento, tipo de cultivo sembrado, manejo del gel y utilización de estabilizantes que se deben aplicar en la elaboración del producto sin influir en la estructura natural del yogur. Es importante que los productores conozcan los mecanismos involucrados en la elaboración de yogur y el impacto de los parámetros de procesamiento en el desarrollo de los geles para mejorar la calidad del producto.

\section{Metodología}

Este trabajo consistió en la búsqueda de información sobre la reología del yogur y los efectos de las operaciones unitarias en el procesamiento del yogurt y el uso de aditivos. Se realizó en concordancia con la metodología PRISMA (Preferred reporting items for systematic reviews and meta-analyses) que incluye un diagrama de flujo que integra estrategias de 
búsqueda y selección de estudios, iniciando con la identificación de estudios relevantes hasta su selección final. La guía contiene 27 ítems y consta de 4 fases: Identificación, Cribado, Elección e Inclusión.

\subsection{Identificación}

Para ampliar la búsqueda científica, se recurrió a las principales bases de datos; Science Direct (7) y Google académico (5). Se utilizaron palabras claves mediante criterios de búsqueda avanzada en inglés como: stirred yogurt, rheology, viscosity, stabilisers, fermentation temperature.

\subsection{Cribado}

Se excluyen aquellos artículos científicos que solo estén relacionados a reología de fluidos, que la fecha de publicación no se encuentre en el intervalo de tiempo establecido de año de publicación 2010 hasta el año 2020, que no sean de relevancia, que describan las características reológicas del yogur que no sea elaborado a partir de leche de bovino, aquellos que no traten sobre la reología y proceso de elaboración de yogur batido, así como, aquellos que no contemplen en su estructura las operaciones unitarias que se detallan en la figura 1.

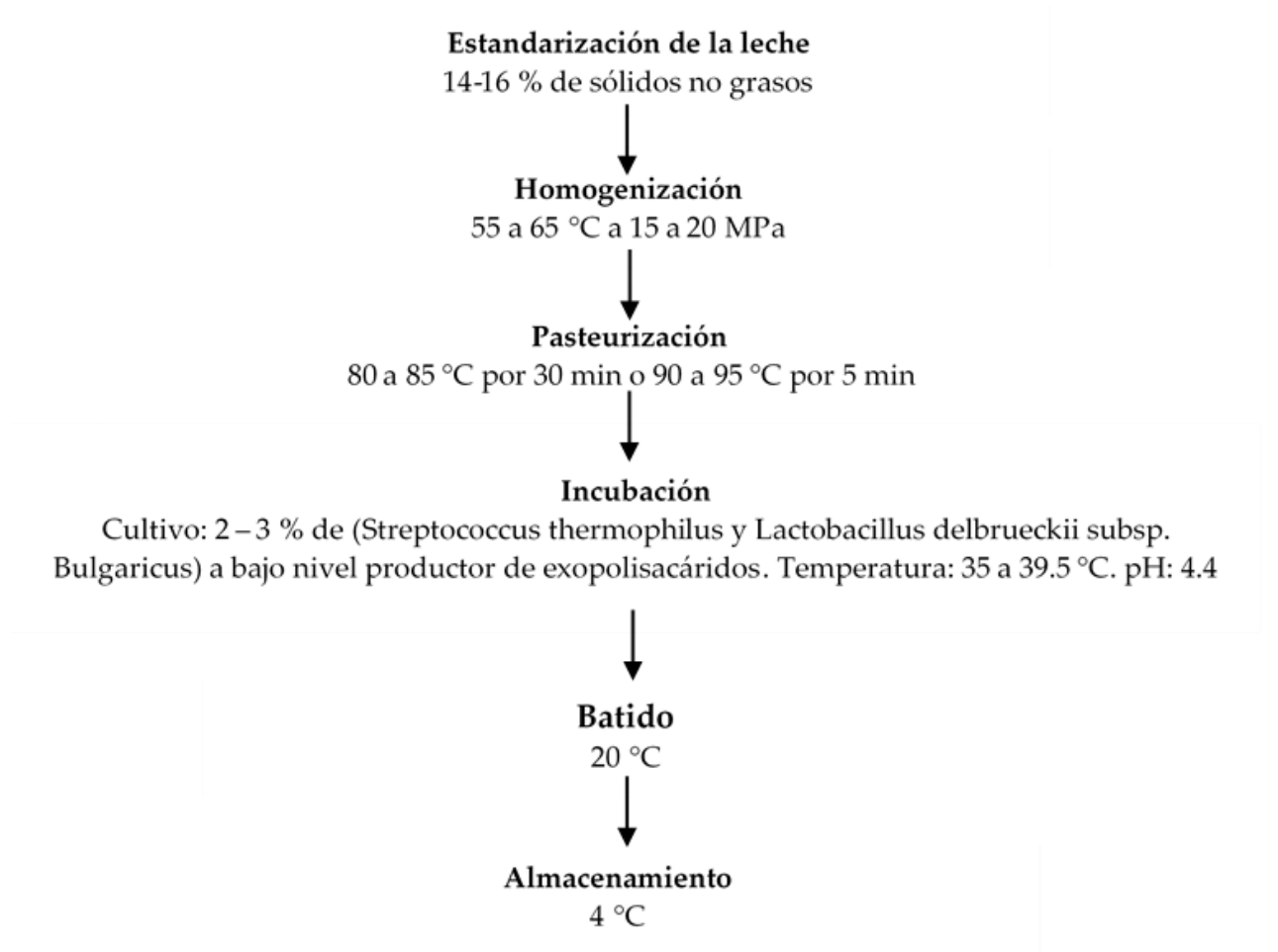

Figura 1: Operaciones unitarias que influyen en la viscosidad del yogur batido. Adaptado de Lee \& Lucey (2010).

\subsection{Elección}

Aquellos artículos que en el título o en el resumen mencione la viscosidad del yogur batido, los factores que afectan la viscosidad, estabilizantes y su efecto en la viscosidad.

\subsection{Inclusión}

Se incluyeron artículos científicos originales y artículos científicos de revisión que tratan sobre las características y propiedades reológicas del yogur batido, operaciones unitarias y su 
influencia en la viscosidad, efecto de los estabilizantes en la viscosidad del yogur batido, en un intervalo de tiempo de publicación del año 2010 hasta el año 2020, aquellos artículos que no se encuentren en el intervalo de tiempo establecido, pero que estén publicados en revistas con factores de impacto y con gran relevancia en el tema y no puedan ser excluidos. Asimismo, se incluyeron artículos científicos de revisión que se enfoquen en la reología del yogur y en las propiedades físicas y químicas del yogur batido.

A continuación, en la figura 2 se detalla el procedimiento de selección de artículos científicos para este estudio mediante el diagrama del método PRISMA.

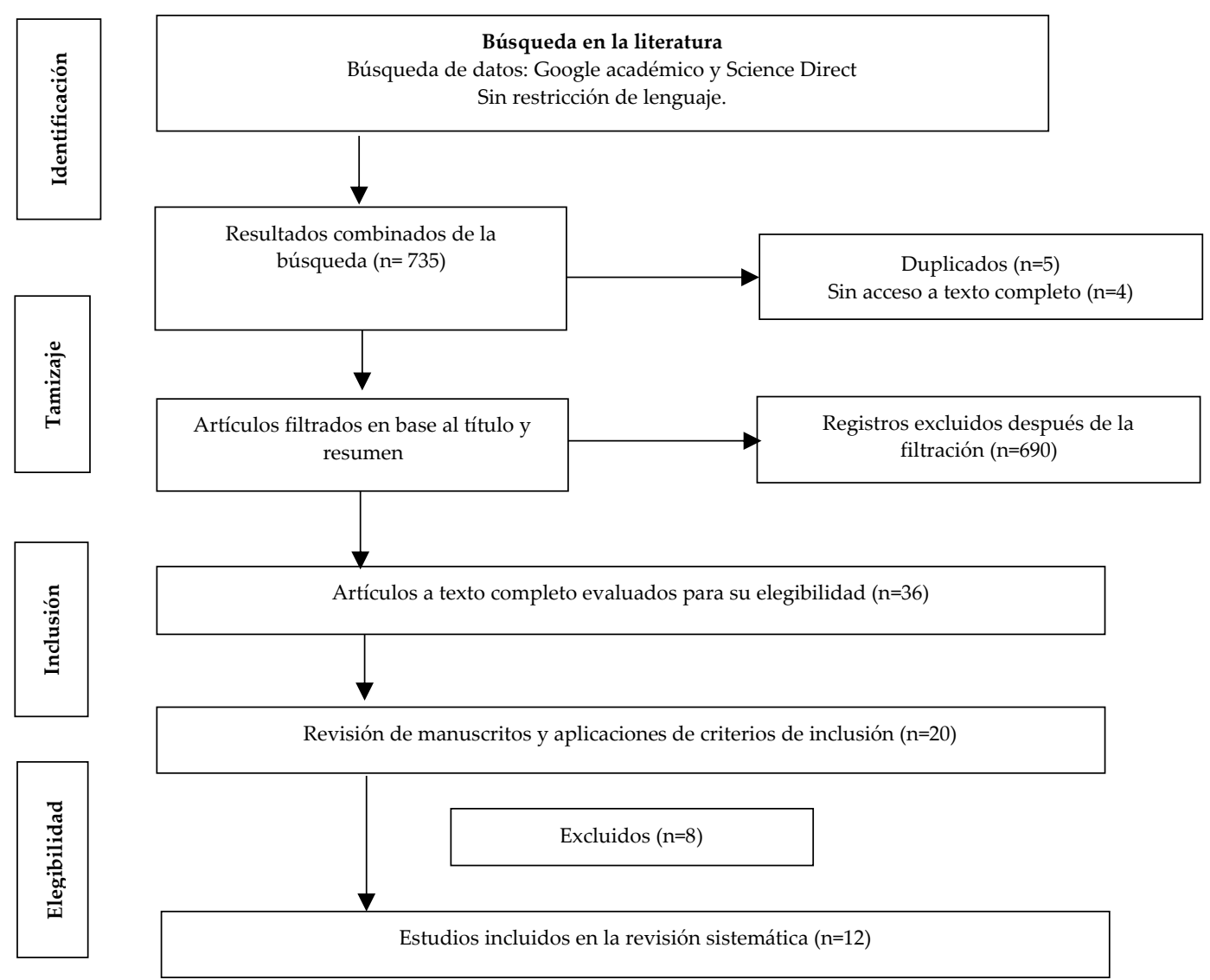

Figura 2: Diagrama método PRISMA: $\mathrm{n}$ = número de artículos en cada una de las etapas hasta su elegibilidad para este estudio.

\section{Resultados}

En la tabla 1 se detallan los factores analizados en los artículos seleccionados que afectan a la viscosidad del yogur batido en las operaciones unitarias durante su elaboración tales como: la estandarización de la leche, temperatura de pasteurización, tipo de cultivo, temperatura de incubación, $\mathrm{pH}$ al que finaliza el proceso de fermentación, temperatura de enfriamiento, batido y temperatura de almacenamiento del yogur. Como se observa en la tabla 1 para la estandarización de la leche el $86 \%$ de los autores utilizaron leche en polvo descremada y el $14 \%$ leche en polvo, por otro lado, para el procesamiento de yogur batido la temperatura de pasteurización utilizada es mayor a $80^{\circ} \mathrm{C}$, además, se evidenció que el rango de temperatura para el proceso de incubación va de $35-45^{\circ} \mathrm{C}$, para el batido cada autor utiliza una metodología diferente en cuanto al equipo, tiempo y velocidad, ocasionando problemas para la comparación de protocolos (Tabla 1). 


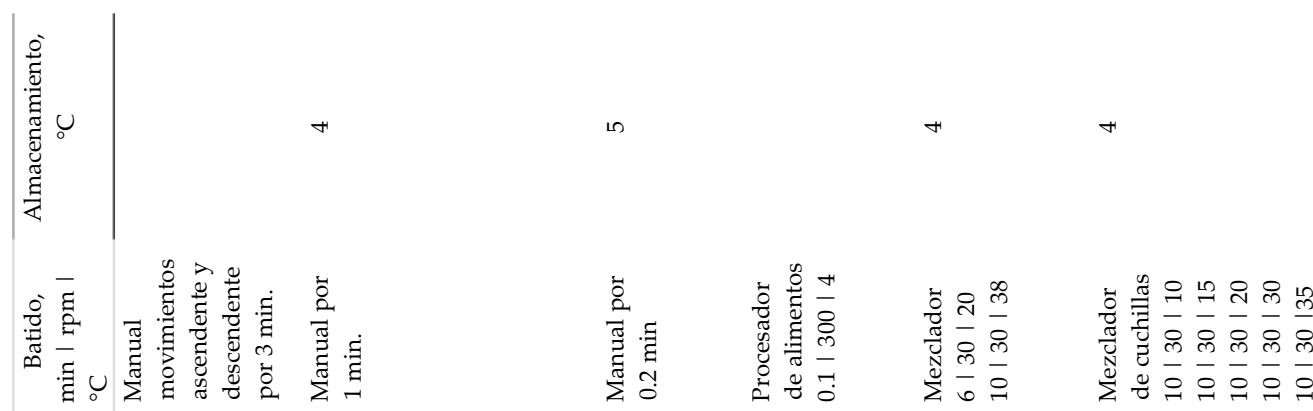

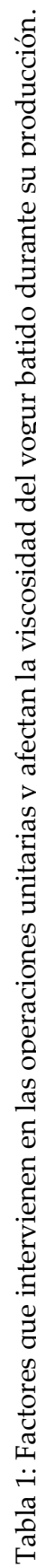

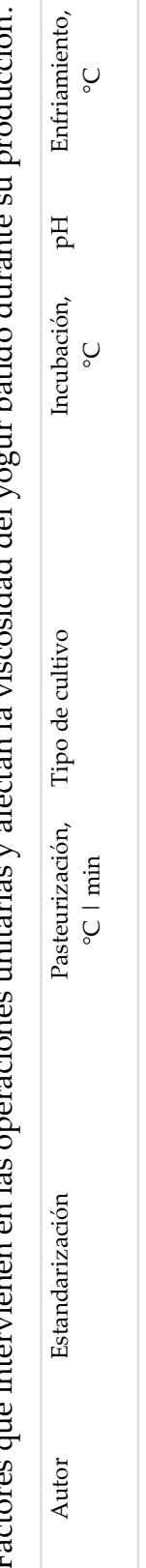

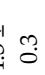

ร

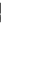

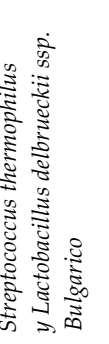

孚

승과 와

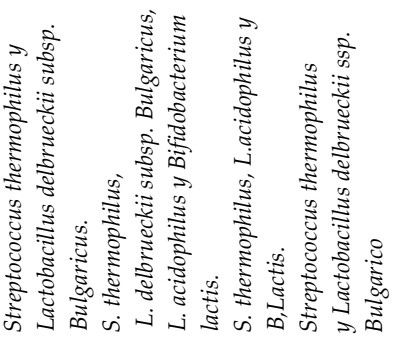

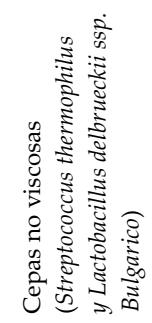

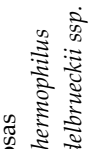

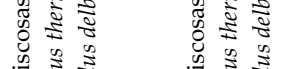

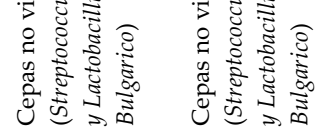

$\frac{10}{\sqrt[2]{2}} \quad \frac{2}{2}$

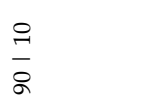

$\frac{10}{10}$

$\frac{10}{\substack{n \\+}}$

$\stackrel{ }{\stackrel{7}{9}}$

0
\[ \]
0
0
+1
$\dot{7}$

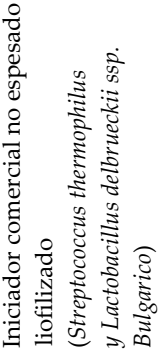

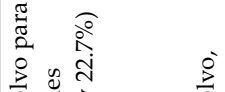

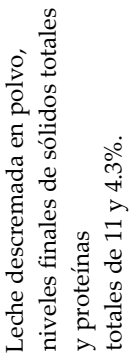

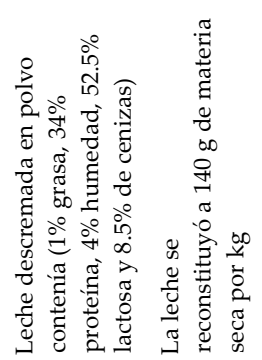

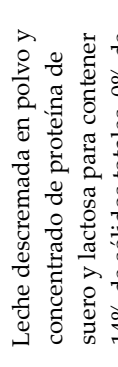

뜬 $\frac{8}{6}$

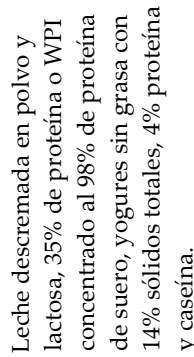

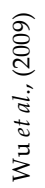

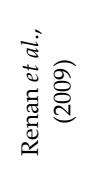

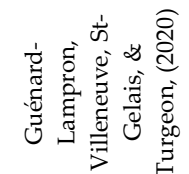

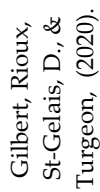
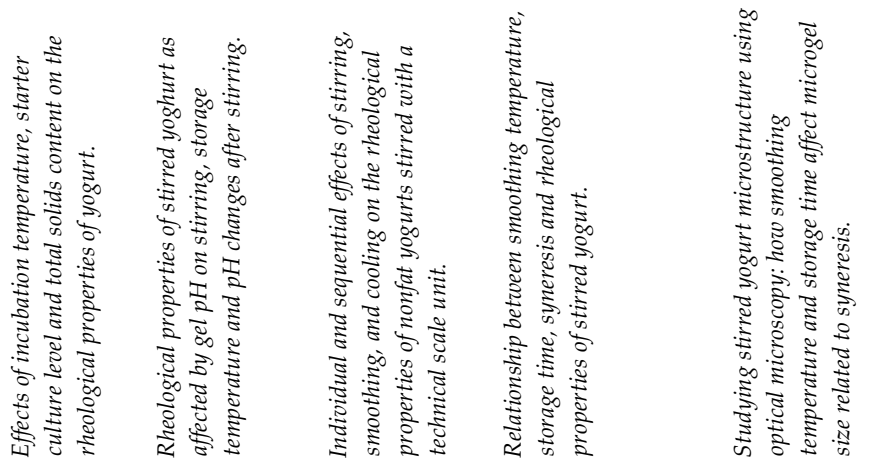
En la tabla 2 se detallan las variables relacionadas con la viscosidad aparente de cada uno de los artículos tales como: equipo utilizado, temperatura de muestra, tiempo y velocidad de corte, que se toman en consideración al realizar el análisis de viscosidad en el yogur batido. De acuerdo con la literatura existen diferentes protocolos para analizar la viscosidad aparente del yogur batido, por lo que resulta complicado realizar un análisis exhaustivo entre los resultados presentados en los estudios seleccionados (Tabla 2).

Tabla 2: Factores y condiciones para el análisis de viscosidad aparente del yogur batido.

\begin{tabular}{|c|c|c|c|c|c|}
\hline Autor & Tema & Equipo & $\begin{array}{l}\text { Temperatura } \\
\text { de la muestra, } \\
{ }^{\circ} \mathrm{C}\end{array}$ & $\begin{array}{l}\text { Tiempo, } \\
\text { min }\end{array}$ & $\begin{array}{c}\text { Velocidad } \\
\text { de corte, } \\
\mathrm{s}^{-1}\end{array}$ \\
\hline $\begin{array}{l}\text { Wu et al., } \\
\text { (2009) }\end{array}$ & $\begin{array}{l}\text { Effects of incubation, } \\
\text { temperature, starter culture level } \\
\text { and total solids content on the } \\
\text { rheological properties of yogurt. }\end{array}$ & $\begin{array}{l}\text { Reómetro (AR-2000ex, } \\
\text { TA Instruments Ltd) } \\
\text { con geometría de } \\
\text { placas paralelas de } \\
\text { aluminio. }\end{array}$ & 5 & 20 & 10 \\
\hline $\begin{array}{l}\text { (Barretto et al., } \\
\text { 2006) }\end{array}$ & $\begin{array}{l}\text { Simultaneous effects of total } \\
\text { solids content, milk base, heat } \\
\text { treatment temperature and } \\
\text { sample temperature on the } \\
\text { rheological properties of plain } \\
\text { stirred yogurt. }\end{array}$ & $\begin{array}{l}\text { Reómetro rotacional } \\
\text { (modelo Rheotest 2.1) } \\
\text { geometría de cilindro } \\
\text { coaxial. }\end{array}$ & $\begin{array}{c}1.6 \\
18.4\end{array}$ & & 100 \\
\hline $\begin{array}{l}\text { Guénard- } \\
\text { Lampron et al., } \\
\text { (2020) }\end{array}$ & $\begin{array}{l}\text { Relationship between smoothing } \\
\text { temperature, storage time, } \\
\text { syneresis and rheological } \\
\text { properties of stirred yogurt. }\end{array}$ & $\begin{array}{l}\text { Reómetro (Reómetro } \\
\text { physical MCR301) }\end{array}$ & 4 & & \\
\hline $\begin{array}{l}\text { Guénard- } \\
\text { Lampron et al., } \\
\text { (2019) }\end{array}$ & $\begin{array}{l}\text { Individual and sequential effects } \\
\text { of stirring, smoothing, and } \\
\text { cooling on the rheological } \\
\text { properties of nonfat yogurts } \\
\text { stirred with a technical scale } \\
\text { unit. }\end{array}$ & $\begin{array}{l}\text { Reómetro (reómetro } \\
\text { physical MCR301) con } \\
\text { cilindro concéntrico. }\end{array}$ & 4 & & 0 a 100 \\
\hline $\begin{array}{l}\text { Renan et al., } \\
\text { (2009) }\end{array}$ & $\begin{array}{l}\text { Rheological properties of stirred } \\
\text { yoghurt as affected by gel } p H \text { on } \\
\text { stirring, storage temperature and } \\
\text { pH changes after stirring. }\end{array}$ & $\begin{array}{l}\text { Viscosímetro VT550 } \\
\text { termostatizado con un } \\
\text { cono de acero. }\end{array}$ & $\begin{array}{c}4 \\
12 \\
20\end{array}$ & 5 & 64 \\
\hline $\begin{array}{l}\text { Guénard- } \\
\text { Lampron et al., } \\
\text { (2020) }\end{array}$ & $\begin{array}{l}\text { Studying stirred yogurt } \\
\text { microstructure using optical } \\
\text { microscopy: How smoothing } \\
\text { temperature and storage time } \\
\text { affect microgel size related to } \\
\text { syneresis. }\end{array}$ & $\begin{array}{l}\text { Reómetro (ARES-G2; } \\
\text { TA instruments) } \\
\text { equipado con una } \\
\text { geometría de copa y } \\
\text { veleta. }\end{array}$ & $\begin{array}{l}42 \\
20\end{array}$ & 5 & 625 \\
\hline
\end{tabular}

\section{Discusión}

4.1. Efecto de las operaciones unitarias en las propiedades reológicas del yogur

\subsubsection{Estandarización}

Barretto et al., (2006) señalaron que en los yogures formulados para contener 10 y $12 \%$ de sólidos no grasos (SNF) la viscosidad no fue significativamente diferente; sin embargo, la viscosidad fue significativamente mayor para los yogures que contenían $14 \%$ de SNF. Según Tamime et al., (1999) la viscosidad mejora cuando el contenido de sólidos totales de la leche aumenta de 12 a $16 \%$. Por otra parte, Wu et al. (2009) lograron mayor viscosidad aparente cuando el contenido de sólidos 
totales fue superior al $15.5 \%$, por lo que podía mejorar la viscosidad aparente del yogur, aumentó de 14.72 Pa a 22.72 Pa cuando el contenido total de sólidos de base de la leche se incrementó de 12.32 \% a $15.68 \%$. Mientras que, Tamime, et al., (1999) incorporaron mayores cantidades de sólidos totales a la leche -mediante el enriquecimiento de contenido de sólidos lácteos no grasos- para lograr un yogur más viscoso, firme y consistente. El análisis de los datos obtenidos por los diferentes autores consultados permite establecer como parámetro de estandarización del contenido de sólidos de la leche bobina entre 14 y16 \%, para alcanzar mayor viscosidad del yogur durante su producción.

\subsubsection{Pasteurización y enfriamiento}

Barretto et al., (2006) observaron que la viscosidad aparente de la leche varía de 22 a $425 \mathrm{mPa}$ al ser sometida a tratamientos térmicos que oscilaron entre 81.6 a $98.4{ }^{\circ} \mathrm{C}$. Así, Barretto et al., (2006) demostrando que la viscosidad aparente y la firmeza de la cuajada estaban altamente correlacionadas con la desnaturalización de las proteínas del suero.

Sin embargo, Küçükçetin et al., (2009) identificaron que el valor del estrés de rendimiento del yogur obtenido de la leche calentada a $95{ }^{\circ} \mathrm{C}$ durante 5 min fue un $37 \%$ mayor que el obtenido de la leche calentada a $130{ }^{\circ} \mathrm{C}$ durante 80 s. Según Mottar, Bassier, Joniau, \& Baert (1989), a una carga de calor más elevada $\left(130^{\circ} \mathrm{C}\right)$ la precipitación de a-lactoalbúmina en una micela da lugar a una reducción hidrofobia superficial y a una superficie micelar más lisa. La pasteurización es una variable de procesamiento importante debido a su influencia sobre las propiedades físicas y estructurales del yogur, durante esta etapa se elimina la mayor cantidad de oxígeno disuelto, lo que ayuda al crecimiento del cultivo iniciador. Además, las temperaturas entre los 80 y $85^{\circ} \mathrm{C}$ por 30 min o 90 y 95 ${ }^{\circ} \mathrm{C}$ por 5 min promueven modificaciones importantes en las proteínas de la leche -caseína y seroproteinas- (Lee \& Lucey, 2010). Por tanto, los autores consultados concuerdan que la desnaturalización parcial de las proteínas del suero tiene una importancia vital en la estabilidad del gel del yogur.

\subsubsection{Tipo de cultivo}

Küçükçetin et al., (2009) obtuvieron valores del módulo de almacenamiento del yogur que evidenciaron un aumento de aproximadamente $40 \%$ en el yogur elaborado con un cultivo iniciador de bajo nivel productor de exopolisacáridos, en lugar de un cultivo alto y medio nivel productor de exopolisacárido. Así, Küçükçetin et al., (2009) demostrando que existe una relación directa entre el número de moléculas que participan en la red tridimensional y la fuerza del gel, especulándose que los cultivos iniciadores productores de exopolisacáridos interfieren con el número y la fuerza de los enlaces entre las partículas de caseína. Wu et al., (2009) demostró que, al utilizarse un cultivo iniciador de alto nivel, la viscosidad aparente del yogur era baja. Mientras que Lee \& Lucey (2010) recomiendan la utilización de un 2 a $3 \%$ de cultivo iniciador de (Streptococcus thermophilus y Lactobacillus delbrueckii subsp. Bulgaricus), estos cultivos son de bajo nivel productor de exopolisacáridos, y no intervienen en la reducción del número de moléculas que generan una red tridimensional más fuerte en el gel, mejorando la viscosidad del yogur.

\subsubsection{Incubación}

Barretto et al., (2006) reportaron un aumento de viscosidad en el yogurt a una temperatura de incubación entre 35 y $39.5^{\circ} \mathrm{C}$. Por otro lado, Küçükçetin et al., (2009) observaron que existe una relación entre el estrés de rendimiento y la temperatura de incubación a $37{ }^{\circ} \mathrm{C}$. Sodini, Remeuf, Haddad, \& Corrieu (2004) observaron el mismo comportamiento a una temperatura entre 42 y 45 ${ }^{\circ} \mathrm{C}$. 
Por otra parte, Wu et al. (2009) reportó que la temperatura de incubación afecta la viscosidad aparente, obteniendo mayores valores de viscosidad aparente a una temperatura de incubación más baja. Debido a que una temperatura más baja provoca una gelificación lenta, es decir las partículas de caseína tendrían mayor volumen y probablemente serían más deformables que a altas temperaturas de incubación, lo que permitía que las partículas se acumulen con un mayor número de enlaces proteína-proteína entre dos partículas diferentes. Lee \& Lucey (2010) demostraron que al utilizar temperaturas menores de incubación $\left(40^{\circ} \mathrm{C}\right)$ y mayor proporción de cultivo iniciador $(3-4$ $\%)$ reduce la sinéresis sobre la superficie del gel.

Lee \& Lucey (2010) concluyeron que temperaturas entre los 40 y $45{ }^{\circ} \mathrm{C}$ son óptimas para el crecimiento y desarrollo de las bacterias termófilas (Streptococcus subsp. thermophilus y Lactobacillus delbrueckii subsp. Bulgaricus) convirtiendo la lactosa en ácido láctico, lo que reduce el $\mathrm{pH}$ de la leche. Demostrando así que la temperatura en esta etapa es muy importante debido a que en esta fase del proceso se determina la estabilidad y componentes del gel.

\subsection{5. $p H$}

Küçükçetin et al., (2009), indicaron que el pH al final del período de incubación fue similar para los diferentes yogures, con un promedio de $\mathrm{pH}$ de 4.4, independientemente del tratamiento aplicado. Los valores de $\mathrm{pH}$ disminuyeron significativamente en cada una de las muestras de yogur después de haber sido almacenados a $4^{\circ} \mathrm{C}$ durante 15 días.

Renan et al., (2009) reportaron que la viscosidad del yogur batido a pH 4.4 es mayor que la del yogur a pH 4.8 (Martin, Skokanova, Latrille, Beal, \& Corrieu, 1998). Este resultado sugiere que variar el pH es una forma eficiente de modificar las propiedades reológicas de los geles. Los resultados presentados confirman que los geles agitados a $\mathrm{pH} 4.4$ que surgieron a partir de geles fijos con los valores $\mathrm{G}$ (módulo elástico) más altos, mostraron el mayor aumento de viscosidad y módulo elástico después de ser agitados. Lee \& Lucey (2010) recomiendan un $\mathrm{pH}<5$ debido a que el punto isoeléctrico de la caseína ( $\mathrm{pH}$ 4.6) conduce a una disminución de la repulsión electrostática entre las moléculas de caseína. Además, las atracciones de caseína-caseína aumentan debido al aumento de las interacciones de carga hidrofóbica. El pH en esta etapa es importante ya que el proceso de acidificación define la formación de una red tridimensional, lo que genera un aumento en la viscosidad después del batido.

\subsubsection{Enfriamiento}

Guénard-Lampron et al. (2019) reportaron que el enfriamiento tiene como objetivo reducir la post acidificación del cultivo iniciador y mejorar las propiedades de textura del yogur (Lucey, 2004). La sinéresis fue menor para el yogur enfriado con intercambiador de calor de placa. No obstante, el tipo de sistema de enfriamiento (intercambiador de placas o tubular) utilizado no afectó los valores de viscosidad, sin embargo, la viscosidad aparente fue mayor en las muestras enfriadas a $20{ }^{\circ} \mathrm{C}$ que a $38^{\circ} \mathrm{C}$. Esta etapa es crítica en la producción de yogur debido a que allí se reduce la actividad metabólica de las bacterias ácido-lácticas para evitar una mayor acidez en el producto.

\subsubsection{Batido o suavizado}

El batido produce un campo de grandes esfuerzos que originan la destrucción de la estructura del gel y la modificación de las propiedades reológicas de los yogures (Zhang, Folkenberg, Amigo, \& Ipsen, 2016). Guénard-Lampron et al., (2019) reportaron que el batido a una temperatura de $20^{\circ} \mathrm{C}$ afecto en mayor medida los valores de viscosidad, sin embargo, se obtuvieron 
valores de sinéresis más bajos para el yogur batido a $10{ }^{\circ} \mathrm{C}$, mientras que temperaturas de batido de 10 a $30{ }^{\circ} \mathrm{C}$ aumentaron los valores de viscosidad. Una observación similar describió Mokoonlall, Nöbel, \& Hinrichs (2016) cuando se batió el yogur a una temperatura de $20^{\circ} \mathrm{C}$. La viscosidad podría aumentar durante el enfriamiento a menor temperatura antes del suavizado y esto puede conducir a una mayor ruptura de la red de proteínas. Gilbert et al. (2020) obtuvo los mismos resultados al suavizar el yogur con un reómetro $42{ }^{\circ} \mathrm{C}$ dando un valor de sinéresis más alto que el yogur suavizado a $20^{\circ} \mathrm{C}$. Diversos autores coinciden en que el batido a $20^{\circ} \mathrm{C}$ es ideal para obtener yogur de alta calidad, que se supone que incluye valores de sinéresis más bajos (Robinson, Lucey, \& Tamime, 2007), debido a que si se suaviza el yogur a una temperatura cercana a la temperatura de incubación dañaría la estructura de la red de proteínas haciéndola más frágil (Lucey, 2004).

Por otra parte, el tamaño del micro gel también puede influir en la viscosidad del yogurt (Mokoonlall et al., 2016), con el cizallamiento intenso y con la formación de pequeños micro geles se produce yogur agitado con baja viscosidad (Mokoonlall et al., 2016) debido a un mayor colapso estructural (Abu-Jdayil, Nasser, \& Ghannam, 2013).

El proceso de batido modifica la estructura coloidal del gel liberando suero y la ruptura de la estructura en frio permite que las micelas de caseína reabsorban el suero evitando la sinéresis por ello se recomienda una temperatura de $20^{\circ} \mathrm{C}$ para el proceso del batido del yogur.

\subsubsection{Almacenamiento}

En el estudio realizado por Guénard-Lampron et al. (2020) encontraron que en la medida que la post acidificación aumentaba -originando la disminución del pH de 4.5 a 4.3- también aumentaron la viscosidad, la firmeza y el tiempo de flujo. Serra, Trujillo, Guamis, \& Ferragut (2009) reportaron de manera similar aumentos en las propiedades reológicas durante el almacenamiento y relacionaron esto con la post acidificación (valor de $\mathrm{pH}$ no especificado). Durante el almacenamiento, la resistencia al flujo y la sinéresis se mantuvieron relativamente estables y no fueron muy sensibles al tiempo de almacenamiento en comparación con las otras propiedades.

A temperaturas de almacenamiento inferiores a $10{ }^{\circ} \mathrm{C}$ se hacen[as lentas las reacciones bioquímicas y biológicas que son resultado de la actividad metabólica de los cultivos del yogur, al reducir la temperatura de almacenamiento a $4{ }^{\circ} \mathrm{C}$ se reduce al mínimo las reacciones, permitiendo conservar la calidad del producto después de su fabricación. Durante las primeras 24 a 48 horas de almacenamiento en refrigeración se observa una mejora de las características físicas del coágulo (Tamime et al.,1999). Razones, por lo que se considera una temperatura menor para el almacenamiento del yogur.

\subsection{Efecto de los aditivos utilizados en las propiedades reológicas del yogur}

\subsubsection{Pectina de bajo metoxi}

Everett \& McLeod (2005) concluyeron que la unión de los agregados de caseína hasta $1 \mathrm{~g}$ de pectina puede fortalecer la red de caseína, reduciendo así la capacidad de retención de agua, pero no afecta la viscosidad en gran medida si la fracción de volumen no cambia. El aumento de la concentración de pectina dio como resultado una disminución en viscosidad aparente y módulos dinámicos y un aumento en la capacidad de retención de agua. A niveles más altos de pectina, las unidades de flujo están cada vez más cubiertas por este polisacárido y los agregados están parcialmente estabilizados estéricamente, lo que conduce a reducir la viscosidad y aumentar la capacidad de retención de agua, a medida que la red de caseína comienza a perder integridad estructural y expulsa la fase sérica. A concentraciones altas de pectina, el yogur no puede dispersarse 
por completo, ya que no se formarían agregados micelares y no se produciría la sinéresis, proporcionando una red más líquida como con más de 2 g pectina.

\subsubsection{Carragenina}

Everett \& McLeod (2005) encontraron un comportamiento similar para este polisacárido hasta con $3 \mathrm{~g} \mathrm{~L}^{-1}$ de estabilizador. La reducción de la viscosidad aparente por los módulos dinámicos y el aumento de la capacidad de retención de agua hasta $3 \mathrm{~g} \mathrm{~L}^{-1}$ de carragenina pueden ocurrir por un mecanismo de estabilización estérica parcial con agregación residual. Los agregados pueden ser unidos por el polisacárido en concentraciones bajas, aunque esto no es concluyente a partir de los datos de capacidad de retención de agua, que no disminuyeron hasta $1 \mathrm{~g} \mathrm{~L}^{-1}$ de carragenina. La unión de las micelas de caseína con las de carragenina, es posible en concentraciones inferiores o alrededor de $1 \mathrm{~g} \mathrm{~L}^{-1}$ a $25^{\circ} \mathrm{C}$ y la floculación por agotamiento puede tener lugar en alrededor de $2 \mathrm{~g}$ $\mathrm{L}^{-1}$ a $65^{\circ} \mathrm{C}$ (Langendorff, Cuvelier, Launay, \& Parker, 1997).

\subsubsection{Goma de guar}

Everett \& McLeod (2005) indicaron que a medida que la concentración de goma-guar aumenta a $1 \mathrm{~g} \mathrm{~L}^{-1}$, la estructura del agregado micelar se vuelve más compacta debido a la floculación por agotamiento. La fracción de volumen resultante redujo la viscosidad aparente, provocando que el yogur sea más líquido. Una estructura agregada más compacta tendrá una menor densidad de enlaces cruzados agregados y, por lo tanto, menor elasticidad (van Vliet, van Dijk, Zoon, \& Walstra, 1991) y un mayor nivel de sinéresis. La goma-guar puede formar una fase continua viscosa a una concentración de $5 \mathrm{~g} \mathrm{~L}^{-1}$ que contiene agregados micelares compactos atrapados. Esto daría como resultado una viscosidad más alta y una capacidad de retención de agua reducida, afectando la viscosidad del yogur.

\subsection{Mediciones Reológicas.}

Wu et al. (2009) concluyó que el reómetro es una herramienta excelente y conveniente en el estudio a profundidad de la estructura del gel en productos viscosos. El uso ya sea del viscosímetro y/o reómetro dependerá del tipo de análisis y la complejidad que se desee llevar.

Con relación a la temperatura de las muestras, Barretto et al., (2006) mencionan que la temperatura comprendida entre 1.6 y $18.4{ }^{\circ} \mathrm{C}$ influye en las propiedades reológicas, al aumentar la temperatura de las muestras, disminuye el índice de consistencia y aumenta el índice de comportamiento del flujo. Mientras que Guénard-Lampron et al., (2019) no observaron diferencias significativas en la viscosidad entre las muestras de yogur tomadas al final del proceso a temperatura de $4{ }^{\circ} \mathrm{C}$ en rangos de velocidades de 0 a $100 \mathrm{~s}^{-1}$.

Sin embargo, Basiri, Haidary, Shekarforoush, \& Niakousari, (2018) observaron que el cambio de viscosidad durante el tiempo de cizallamiento en diferentes tratamientos está influenciado por el aumento de velocidad y el tiempo de rotación del rotor (spindle) en el viscosímetro, la viscosidad aparente disminuye en todas las muestras durante el cizallamiento durante todos los días de evaluación.

Algunos autores no tomaron en cuenta las características de la muestra y las velocidades del rotor como factores que influencian la viscosidad de las muestras analizadas, solo se basaron en intervalos de velocidades de 0 a $100 \mathrm{~s}^{-1}$, con temperaturas comprendidas desde los $4{ }^{\circ} \mathrm{C}$ hasta $\operatorname{los} 20^{\circ} \mathrm{C}$. 


\section{Conclusiones}

La viscosidad es influenciada por las características físico-químicas de la leche y operaciones unitarias presentes en el procesamiento de yogur. Los emulsionantes y estabilizantes influyen directamente en la viscosidad del producto final contribuyendo al aumento de la sinéresis del yogur durante el tiempo de almacenamiento.

Investigaciones futuras para confirmar estos hallazgos se deberían realizar ya que existen pocos trabajos en la literatura acerca de los protocolos para determinar la viscosidad del yogur, en los cuales no se detallan con exactitud, variables como la temperatura de la muestra y rangos de velocidades de corte.

\section{Contribuciones de los autores}

En concordancia con la taxonomía establecida internacionalmente para la asignación de créditos a autores de artículos científicos (https://casrai.org/credit/). Los autores declaran sus aportes en la siguiente matriz de contribuciones:

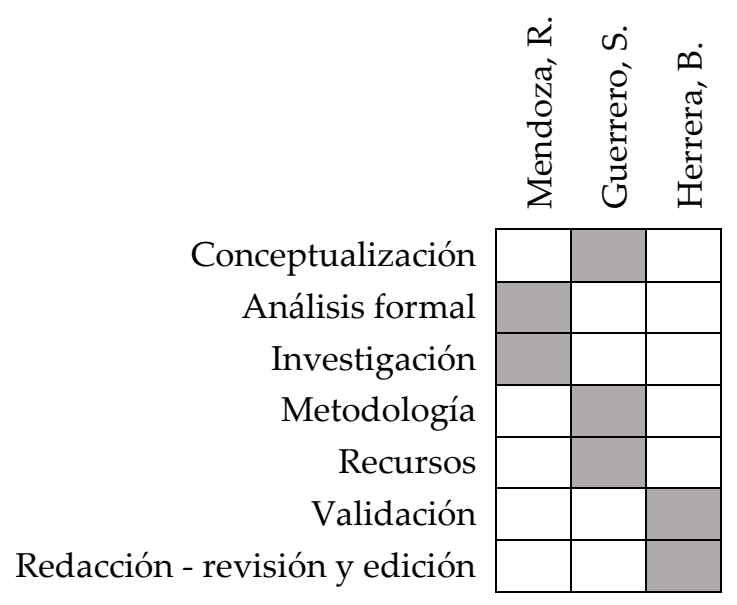

\section{Conflictos de Interés}

Los autores declaran que han leído y están de acuerdo con la versión publicada del manuscrito.

\section{Referencias}

Abu-Jdayil, B., Nasser, M. S., \& Ghannam, M., (2013). Structure breakdown of stirred yoghurt in a circular pipe as affected by casein and fat content. Food Science and Technology Research, 19(2), 277-286. https://doi.org/10.3136/fstr.19.277

Afonso, I. M., \& Maia, J. M. (1999). Rheological monitoring of structure evolution and development in stirred yoghurt. Journal of Food Engineering, 42(4), 183-190. https://doi.org/10.1016/S0260-8774(99)00118-1

Barretto, A. L., Converti, A., \& Nogueira, M., (2006). Simultaneous effects of total solids content, milk base, heat treatment temperature and sample temperature on the rheological properties of plain stirred yogurt. Food Technology and Biotechnology, 44(4), 515-518. Recuperado de https://hrcak.srce.hr/index.php?show=clanak\&id_clanak_jezik=162423

Basiri, S., Haidary, N., Shekarforoush, S. S., \& Niakousari, M. (2018). Flaxseed mucilage: A natural stabilizer in stirred yogurt. Carbohydrate Polymers, 187, 59-65. https://doi.org/10.1016/j.carbpol.2018.01.049 
O'Donnell, H. J. \& Butler, F. (2002). Time-dependent viscosity of stirred yogurt. Part I: couette flow. Journal of Food Engineering, 51(3), 249-254. https://doi.org/10.1016/S0260-8774(01)00064-4

Everett, D. W., \& McLeod, R. E. (2005). Interactions of polysaccharide stabilisers with casein aggregates in stirred skim-milk yoghurt. International Dairy Journal, 15(11), 1175-1183. https://doi.org/10.1016/j.idairyj.2004.12.004

Gilbert, A., Rioux, L. E., St-Gelais, D., \& Turgeon, S. L. (2020). Studying stirred yogurt microstructure using optical microscopy: How smoothing temperature and storage time affect microgel size related to syneresis. Journal of Dairy Science, 103(3), 2139-2152. https://doi.org/10.3168/jds.2019-16787

Guénard-Lampron, V., Villeneuve, S., St-Gelais, D., \& Turgeon, S. L. (2020). Relationship between smoothing temperature, storage time, syneresis and rheological properties of stirred yogurt. International Dairy Journal, 109, 104742. https://doi.org/10.1016/j.idairyj.2020.104742

Guénard-Lampron, V., St-Gelais, D., Villeneuve, S., \& Turgéon, S. L., (2019). Individual and sequential effects of stirring, smoothing, and cooling on the rheological properties of nonfat yogurts stirred with a technical scale unit. Journal of Dairy Science, 102(1), 190-201. https://doi.org/10.3168/jds.2018-14565

Küçükçetin, A., Weidendorfer, K., \& Hinrichs, J. (2009). Graininess and roughness of stirred yoghurt as influenced by processing. International Dairy Journal, 19(1), 50-55. https://doi.org/10.1016/j.idairyj.2008.07.006

Langendorff, V., Cuvelier, G., Launay, B., \& Parker, A. (1997). Gelation and flocculation of casein micelle/carrageenan mixtures. Food Hydrocolloids, 11(1), 35-40. https://doi.org/10.1016/S0268005X(97)80008-2

Lee, W. J., \& Lucey, J. A. (2010). Formation and physical properties of yogurt. Asian-Australasian Journal of Animal Sciences, 23(9), 1127-1136. https://doi.org/10.5713/ajas.2010.r.05

Lucey, J. A. (2004). Cultured dairy products: an overview of their gelation and texture properties. International Journal of Dairy Technology, 57(1-2), 77-84. https://doi.org/10.1111/j.1471-0307.2004.00142.x

Martin, N. C., Skokanova, J., Latrille, E. F. J., Beal, C. E., \& Corrieu, G. V. (1998). Sensory and instrumental characterization of the texture of stirred yoghurt. In International Dairy Federation Symposium. (pp. 2433). Vicenza, Italy. https://hal.inrae.fr/hal-02770251

Molina, I. S. (2009). Comparación de tres estabilizantes comerciales utilizados en la elaboración de yogurt de leche descremada de vaca (Tesis de Licenciatura). Ciudad de Guatemala Guatemala: Universidad de San Carlos de Guatemala. http://www.repositorio.usac.edu.gt/3261/

Mokoonlall, A., Nöbel, S., \& Hinrichs, J. (2016). Post-processing of fermented milk to stirred products: Reviewing the effects on gel structure. Trends in Food Science $\mathcal{E}$ Technology, 54, 26-36. https://doi.org/10.1016/j.tifs.2016.05.012

Mottar, J., Bassier, A., Joniau, M., \& Baert, J. (1989). Effect of heat-induced association of whey proteins and casein micelles on yogurt texture. Journal of Dairy Science, 72(9), 2247-2256. https://doi.org/10.3168/jds.S0022-0302(89)79355-3

Renan, M., Guyomarch, F., Arnoult-Delest, V., Paquet, D., Brulé, G. \& Famelart, M. (2009). Rheological properties of stirred yoghurt as affected by gel $\mathrm{pH}$ on stirring, storage temperature and $\mathrm{pH}$ changes after stirring. International Dairy Journal, 19(3), 142-148. https://doi.org/10.1016/j.idairyj.2008.09.007

Robinson, R. K., Lucey, J. A., \& Tamime, A. Y. (2007). Manufacture of yoghurt. In A. Tamime (Ed.), A. Fermented Milks (pp. 53-75). Oxford, UK: Blackwell Science Ltd. https://doi.org/10.1002/9780470995501.ch3

Serra, M., Trujillo, A. J., Guamis, B., \& Ferragut, V. (2009). Evaluation of physical properties during storage of set and stirred yogurts made from ultra-high-pressure homogenization treated milk. Food Hydrocolloids, 23(1), 82-91. https://doi.org/10.1016/j.foodhyd.2007.11.015 
Wu, S., Li, D., Li, S., Bhandari, B., Yang, B., Chen, X. D., \& Mao, Z., (2009). Effects of incubation temperature, starter culture level and total solids content on the rheological properties of yogurt. International Journal of Food Engineering, 5(2). https://doi.org/10.2202/1556-3758.1436

Sodini, I., Remeuf, F., Haddad, S., \& Corrieu, G. (2004). The relative effect of milk base, starter, and process on yogurt texture: A review. Critical Reviews in Food Sciences and Nutrition, 44(2), 113-137. https://doi.org/10.1080/10408690490424793

Soukoulis, C., Panagiotidis, P., Koureli, R., \& Tzia, C. (2007). Industrial yogurt manufacture: monitoring of fermentation process and improvement of final product quality. Journal of Dairy Science, 90(6), 26412654. https://doi.org/10.3168/jds.2006-802

Tamime, A. Y., Davies, G., Chehade, A. S., \& Mahdi, H. A. (1989). The production of 'Labneh' by ultrafiltration: a new technology. Journal of the Society of Dairy Technology, 42(2), 35. https://doi.org/10.1111/j.14710307.1989.tb02150.x

Tamime, A. Y., Muir, D. D. \& Wszolek, M. (1999). Kefir, koumiss and kishk. Dairy Industries International, 64(5), 32-33. http://pascal-francis.inist.fr/vibad/index.php?action=getRecordDetail\&idt=1889760

van Vliet, T., van Dijk, H. J. M., Zoon, P., \& Walstra, P. (1991). Relation between syneresis and rheological properties of particle gels. Colloid and Polymer Science, 269, 620-627. https://doi.org/10.1007/BF00659917

Vedamuthu, E. R. (1991). The yogurt story-past, present and future. Recuperado de: https://agris.fao.org/agrissearch/search.do?recordID=US9191369 .

Vélez-Ruiz, J. F., Barbosa, G. V., \& Peleg, M., (1997). Rheological properties of selected dairy products. Critical Reviews in Food Science and Nutrition, 37(4),311-359. https://doi.org/10.1080/10408399709527778

Zhang, L., D. Folkenberg, M. Amigo, J. M., \& Ipsen, R., (2016). Effect of exopolysaccharide-producing starter cultures and post-fermentation mechanical treatment on textural properties and microstructure of low-fat yoghurt. International Dairy Journal, 53:10-19. https://doi.org/10.1016/j.idairyj.2015.09.008 